\title{
A!
}

This is an electronic reprint of the original article.

This reprint may differ from the original in pagination and typographic detail.

Salo, J.; Hekking, Frank; Pekola, Jukka

\section{Frequency-dependent current correlation functions from scattering theory}

Published in:

Physical Review B

DOI:

10.1103/PhysRevB.74.125427

Published: 29/09/2006

Document Version

Publisher's PDF, also known as Version of record

Please cite the original version:

Salo, J., Hekking, F., \& Pekola, J. (2006). Frequency-dependent current correlation functions from scattering theory. Physical Review B, 74(12), 1-13. [125427]. https://doi.org/10.1103/PhysRevB.74.125427

This material is protected by copyright and other intellectual property rights, and duplication or sale of all or part of any of the repository collections is not permitted, except that material may be duplicated by you for your research use or educational purposes in electronic or print form. You must obtain permission for any other use. Electronic or print copies may not be offered, whether for sale or otherwise to anyone who is not an authorised user. 


\title{
Frequency-dependent current correlation functions from scattering theory
}

\author{
J. Salo \\ Laboratory of Physics, Helsinki University of Technology, P.O. Box 4100, 02015 TKK, Finland
}

F. W. J. Hekking

Laboratoire de Physique et Modélisation des Milieux Condensés, CNRS \& Université Joseph Fourier, Boîte Postal 166, 38042 Grenoble cedex 09, France

J. P. Pekola

Low Temperature Laboratory, Helsinki University of Technology, P.O. Box 3500, 02015 TKK, Finland

(Received 2 June 2006; revised manuscript received 3 August 2006; published 29 September 2006)

\begin{abstract}
We present a general formalism based on scattering theory to calculate quantum correlation functions involving several time-dependent current operators. A key ingredient is the causality of the scattering matrix, which allows one to deal with arbitrary correlation functions. The formalism proves useful, e.g., in view of recent developments in full counting statistics of charge transfer, where detecting schemes have been proposed for measurement of frequency dependent spectra of higher moments. Some of these schemes are different from the well-known fictitious spin detector and therefore generally involve calculation of non-Keldysh-contourordered correlation functions. As an illustration of the approach we consider various third order correlation functions of current, including the usual third cumulant of current statistics. We investigate the frequency dependence of these correlation functions explicitly in the case of energy-independent scattering. The results can easily be generalized to the calculation of arbitrary $n$th order correlation functions, or to include the effect of interactions.
\end{abstract}

DOI: 10.1103/PhysRevB.74.125427

\section{INTRODUCTION}

Dynamical noise properties of mesoscopic systems have been studied for more than a decade, both theoretically and experimentally. ${ }^{1}$ By now it is well understood that noise measurements can reveal information on the system that is not contained in its dc conductance. So far, most experiments concentrated on measurement of zero-frequency noise. However, several proposals have considered the possibility of detecting finite-frequency noise, for instance through emission and absorption measurements using quantum few level systems such as quantum dots ${ }^{2}$ or small Josephson junctions ${ }^{3}$ as noise detectors. Successful experiments of this type have been reported recently. ${ }^{4,5}$ Finite-frequency noise is interesting, first of all as one expects the noise to probe the intrinsic dynamics of the conductor and hence the noise spectral function should be sensitive to the dwell time $\tau_{D}$ of the carriers. Second, at finite frequency, current is no longer spatially homogeneous, and charge piles up in the conductor. Coulomb interaction screens this pileup of charge, at a characteristic charge relaxation frequency $1 / \tau$ which may well be different from $1 / \tau_{D}$. These issues have been studied theoretically for diffusive contacts in Refs. 6 and 7. Recent calculations of current noise in chaotic cavities ${ }^{8,9}$ that take both the energydependence of scattering and Coulomb interactions into account show that the frequency-dependent noise spectrum is determined solely by the time $\tau$, as long as quantum corrections such as weak-localization can be ignored.

In view of recent interest in the theory of the full counting statistics (FCS) of charge transfer, ${ }^{10}$ attention shifted from the conventional noise to the study of the properties of the higher moments. Recent measurements have probed the zero-frequency third cumulant. ${ }^{11-13}$ As far as the frequency dependence of the higher cumulants is concerned, the situa-
PACS number(s): 72.10.Bg, 72.70. $+\mathrm{m}, 73.23 .-\mathrm{b}$

tion changes drastically as compared to conventional noise spectra. Calculations of the frequency-dependent third cumulant for a chaotic cavity ${ }^{8}$ and for a diffusive conductor ${ }^{14}$ show marked differences from the conventional noise: it is not only determined by the charge-relaxation time $\tau$ but also shows a low-frequency dispersion that is determined by the dwell time $\tau_{D}$.

A properly designed experiment, capable of measuring the frequency-dependent third cumulant, would thus enable one to determine the two relevant time scales separately in a mesoscopic conductor. The question as to how to design such an experiment brings us to one of the key problems of this field: what is an adequate detector to measure frequencydependent noise spectra, and which noise spectral function is it actually measuring? Most of the applications of FCS discussed so far concentrate on the use of a fictitious spin detector, introduced by Levitov and co-workers: ${ }^{15,16}$ the current measured couples to the coherences (off-diagonal elements) of the spin density operator, and the detector responds to the Keldysh contour-ordered correlation functions. Powerful theoretical tools have been developed to calculate these correlation functions; therefore this detector is amenable to straightforward analysis. However, the spin detector might not be the most suitable one for detecting finite frequency noise. Detectors that interact with the noise source through emission and absorption, such as the above mentioned quantum dot and Josephson junction devices, might be more suitable for this task. The measured spectra are then not directly related to Keldysh-ordered correlation functions, and different methods are required to determine these spectra theoretically.

In this paper we develop a method capable of handling arbitrarily ordered correlation functions at both zero and finite temperatures. The formalism we adopt is based on scat- 
tering theory in the absence of interactions, ${ }^{17}$ pioneered in Refs. 18-20. It is the natural approach to discuss transport and noise in mesoscopic devices. The operator for electric current $\hat{I}$ is written as the difference between the current carried by incident particles $\hat{I}_{\text {in }}$ and the current carried by scattered particles $\hat{I}_{\text {out }}: \hat{I}=\hat{I}_{\text {in }}-\hat{I}_{\text {out }}$. The central quantity of the scattering approach is the energy-dependent scattering matrix. It must satisfy the causality condition in real-time representation, which has immediate consequences for the commutation relations between the operators $\hat{I}_{\text {in }}$ and $\hat{I}_{\text {out }}$ at different times. ${ }^{21}$ As a result, any (anti) time-ordered product of current operators can be conveniently rewritten as products of currents $\hat{I}_{\text {in }}$ and $\hat{I}_{\text {out }}$ with all in-currents ordered to the right (left) of the out currents. Denoting (anti) time ordering by $T(\widetilde{T})$ this implies $T\left[\hat{I}_{\text {in }}\left(t_{1}\right) \hat{I}_{\text {out }}\left(t_{2}\right)\right]=\hat{I}_{\text {out }}\left(t_{2}\right) \hat{I}_{\text {in }}\left(t_{1}\right)$ and $\widetilde{T}\left[\hat{I}_{\text {in }}\left(t_{1}\right) \hat{I}_{\text {out }}\left(t_{2}\right)\right]=\hat{I}_{\text {in }}\left(t_{1}\right) \hat{I}_{\text {out }}\left(t_{2}\right)$ independent of the ordering of $t_{1}$ and $t_{2}$. This way, the cumbersome time ordering can be avoided and the remaining in-out-ordered products can be readily calculated using the scattering theory.

We apply the in-out-ordering method to the well-studied case of the third cumulant of charge transfer in a mesoscopic conductor. We treat energy-independent scattering, and present the time-dependent cumulant in the cases of a tunnel barrier (a quantum point contact), a diffusive wire, and a chaotic cavity. First of all, this enables a direct check on the validity of the approach. Second, we believe that the zero frequency limit of the calculation provides a demonstration of the validity of the result for the third cumulant of a tunnel barrier presented in Ref. 22. This issue has given rise to some discussion in the literature, ${ }^{15,16,23}$ and methods have been developed to settle the frequency-dependent case..$^{24,25}$ Thirdly, calculation of the frequency-dependent third cumulant can be used to obtain the asymptotic time dependence of the third cumulant of the charge transfer, both in the short and the long time limits.

The paper is organized as follows. We first summarize the scattering formalism in order to define the notation used later, and use the causality of the scattering matrix to derive important commutation relations between in- and out-current operators. They are used to establish operator transformation rules, such as $T\left[\hat{I}_{\text {in }}\left(t_{1}\right) \hat{I}_{\text {out }}\left(t_{2}\right)\right]=\hat{I}_{\text {out }}\left(t_{2}\right) \hat{I}_{\text {in }}\left(t_{1}\right)$, which allow one to resolve time-ordered products of currents in terms of inout-ordered products. Their main application is to find multicurrent correlation functions, and we explicitly present all three-current correlations, which are written in terms of three-current spectral functions of two frequency arguments. To keep the presentation transparent, we do not address here issues concerning the finite dwell time of carriers nor do we address interaction effects. We thus treat the case of energyindependent scattering where the various spectral functions can be evaluated using only the transmission probabilities of the scatterer, valid in the limit where the abovementioned characteristic times $\tau_{D}, \tau$ vanish. It is important to note that, even though we neglect the energy dependence of the scattering matrix, we do respect its causality through the in-outordering properties. We finally discuss several different detection schemes, which all correspond to different threecurrent correlation functions and, most importantly, use the

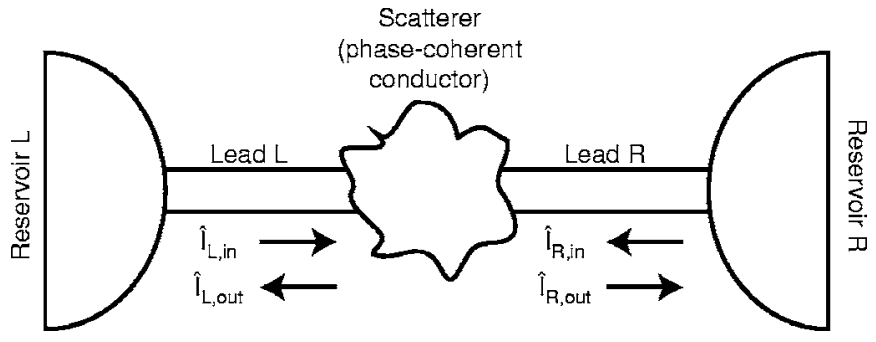

FIG. 1. Two-terminal scattering problem. Both reservoirs are assumed to be in thermal equilibrium, characterized by a common temperature $T$ and potentials such that $V_{R}-V_{L}=V$.

full-counting statistics approach to derive an expression to the time-dependent third cumulant of transmitted charge distribution.

\section{SCATTERING FORMALISM AND CAUSALITY}

\section{A. Scattering theory}

The starting point for the analysis is scattering theory, as developed by Büttiker. ${ }^{17}$ In this formalism, the current operator of noninteracting electrons is given by

$$
\begin{aligned}
\hat{I}_{\alpha}(t)= & \frac{e}{h} \sum_{n} \int d E d E^{\prime} e^{i\left(E-E^{\prime}\right) t / \hbar}\left[\hat{a}_{\alpha n}^{\dagger}(E) \hat{a}_{\alpha n}\left(E^{\prime}\right)\right. \\
& \left.-\hat{b}_{\alpha n}^{\dagger}(E) \hat{b}_{\alpha n}\left(E^{\prime}\right)\right] .
\end{aligned}
$$

The operators $\hat{a}_{\alpha n}^{\dagger}(E)$ and $\hat{a}_{\alpha n}(E)$ create and annihilate electrons with total energy $E$ in the transverse channel $n$ in lead $\alpha$, incident upon the scatterer. Similarly, the creation $\hat{b}_{\alpha n}^{\dagger}(E)$ and annihilation $\hat{b}_{\alpha n}(E)$ operators refer to electrons in the outgoing states. For the two-terminal setup depicted in Fig. 1, $\alpha$ takes values $L$ and $R$ for the left and right leads, respectively. The results to be presented below can be easily generalized to any multiterminal case. The creation and annihilation operators obey the anticommutation relations, for instance,

$$
\begin{gathered}
\hat{a}_{\alpha n}^{\dagger}(E) \hat{a}_{\alpha n^{\prime}}\left(E^{\prime}\right)+\hat{a}_{\alpha n^{\prime}}\left(E^{\prime}\right) \hat{a}_{\alpha n}^{\dagger}(E)=\delta_{n n^{\prime}} \delta\left(E-E^{\prime}\right), \\
\hat{a}_{\alpha n}^{\dagger}(E) \hat{a}_{\alpha n^{\prime}}^{\dagger}\left(E^{\prime}\right)+\hat{a}_{\alpha n^{\prime}}^{\dagger}\left(E^{\prime}\right) \hat{a}_{\alpha n}^{\dagger}(E)=0, \\
\hat{a}_{\alpha n}(E) \hat{a}_{\alpha n^{\prime}}\left(E^{\prime}\right)+\hat{a}_{\alpha n^{\prime}}\left(E^{\prime}\right) \hat{a}_{\alpha n}(E)=0 .
\end{gathered}
$$

Similar anticommutation relations hold naturally also for operators referring to the outgoing states.

The operators $\hat{a}$ and $\hat{b}$ are related by the scattering matrix $s$

$$
\hat{b}_{\alpha n}(E)=\sum_{\beta, m} s_{\alpha \beta ; n m}(E) \hat{a}_{\beta m}(E)
$$

and the creation operators $\hat{a}^{\dagger}$ and $\hat{b}^{\dagger}$ are correspondingly related by the Hermitian conjugated matrix $s_{\alpha \beta ; n m}^{\dagger}(E)$ $=s_{\alpha \beta ; m n}^{*}(E)$. The matrix $s$ is quite generally unitary and it has dimensions $\left(N_{L}+N_{R}\right) \times\left(N_{L}+N_{R}\right)$. Its size and the matrix elements depend on the total energy $E$. It has the block structure 


$$
s=\left(\begin{array}{cc}
r & t^{\prime} \\
t & r^{\prime}
\end{array}\right) .
$$

Electron reflection back to the left and right reservoirs is described by the square diagonal blocks $r$ (size $N_{L} \times N_{L}$ ) and $r^{\prime}$ (size $N_{R} \times N_{R}$ ), respectively, while the off-diagonal, rectangular blocks $t$ (size $N_{R} \times N_{L}$ ) and $t^{\prime}$ (size $N_{L} \times N_{R}$ ) determine, in turn, the electron transmission through the sample.

In order to directly benefit from consequences of causality, we present the current operator as the difference between two directed currents, carried by incoming states and outgoing states, respectively. ${ }^{21}$ Specializing to the left lead, we thus write

$$
\hat{I}_{L}(t)=\hat{I}_{L, \text { in }}(t)-\hat{I}_{L, \text { out }}(t),
$$

where

$$
\hat{I}_{L, \text { in }}(t)=\frac{e}{h} \sum_{n} \int d E d E^{\prime} e^{i\left(E-E^{\prime}\right) t / \hbar} \hat{a}_{L n}^{\dagger}(E) \hat{a}_{L n}\left(E^{\prime}\right)
$$

and

$$
\hat{I}_{L, \text { out }}(t)=\frac{e}{h} \sum_{n} \int d E d E^{\prime} e^{i\left(E-E^{\prime}\right) t / \hbar} \hat{b}_{L n}^{\dagger}(E) \hat{b}_{L n}\left(E^{\prime}\right) .
$$

Now, using Eq. (3) as well as its Hermitian conjugated version, $\hat{I}_{L, \text { out }}(t)$ can also be written as

$$
\begin{aligned}
\hat{I}_{L, \text { out }}(t)= & \frac{e}{h} \sum_{\alpha, \beta} \sum_{m, n, k} \int d E d E^{\prime} e^{i\left(E-E^{\prime}\right) t / \hbar} \hat{a}_{\alpha m}^{\dagger}(E) \\
& \times s_{L \alpha ; m k}^{\dagger}(E) s_{L \beta ; k n}\left(E^{\prime}\right) \hat{a}_{\beta n}\left(E^{\prime}\right),
\end{aligned}
$$

where indices $\alpha$ and $\beta$ may take values $L$ or $R$. This result makes the dependence of the current operator $\hat{I}_{\text {out }}$ on the energy-dependent scattering matrix $s(E)$ explicit. As we will detail below, the commutation properties of directed current operators at different times are completely determined by the analytical properties of $s(E)$.

\section{B. Causality}

In real time, the scattering matrix connects operators of an incoming state with those of an outgoing state by the convolution relation

$$
\hat{b}_{\alpha n}(t)=\sum_{\beta, m} \int_{-\infty}^{\infty} s_{\alpha \beta ; n m}(t-\tau) \hat{a}_{\beta m}(\tau) d \tau .
$$

By causality, the scattering matrix must vanish for negative arguments since otherwise an incident current at $t_{1}$ would cause an outgoing current at $t_{2}<t_{1}$. This is equivalent to requiring that the Fourier transform of the scattering matrix, $s_{\alpha \beta ; n m}(\omega)$ be analytic in the entire upper half plane, since then

$$
s_{\alpha \beta ; n m}(\omega)=\lim _{\eta \rightarrow 0^{+}} \int_{-\infty}^{\infty} \frac{d \omega^{\prime}}{2 \pi i} \frac{s_{\alpha \beta ; n m}\left(\omega^{\prime}\right)}{\left(\omega^{\prime}-\omega\right)-i \eta},
$$

which can be substituted into the inverse Fourier transform of the scattering matrix in order to obtain

$$
\begin{aligned}
s_{\alpha \beta ; n m}(t) & =\int_{-\infty}^{\infty} \frac{d \omega^{\prime}}{2 \pi} s_{\alpha \beta ; n m}\left(\omega^{\prime}\right) \int_{-\infty}^{\infty} \frac{d \omega}{2 \pi i} e^{-i \omega t} \frac{1}{\left(\omega^{\prime}-\omega\right)-i \eta} \\
& =\theta(t) s_{\alpha \beta ; n m}(t) .
\end{aligned}
$$

Hence the analytical structure of $s(\omega)$ as a function of $\omega$ (analyticity in the entire upper half plane) implies causality, ${ }^{26,27}$ i.e., $s\left(t-t^{\prime}\right)=0$ if $t<t^{\prime}$. Similarly, the Hermitian conjugated scattering matrix, $s_{\alpha \beta ; n m}^{\dagger}(\omega)$, must be analytic in the entire lower half plane.

\section{Commutation relations}

We will use the analytical structure of the scattering matrix established in the previous subsection, Eq. (11), to obtain the commutation relations for directed current operators $\hat{I}_{\text {in }}$ and $\hat{I}_{\text {out }}$ at different times. ${ }^{21}$ Consider the commutation relation of $\hat{I}_{L, \text { in }}\left(t_{1}\right)$ and $\hat{I}_{L, \text { out }}\left(t_{2}\right)$. Starting from

$$
\left[\hat{I}_{L, \text { in }}\left(t_{1}\right), \hat{I}_{L, \text { out }}\left(t_{2}\right)\right]=\left(\frac{e}{h}\right)^{2} \sum_{n_{1}, n_{2}} \int d E_{1} d E_{2} d E_{3} d E_{4} e^{i\left(E_{1}-E_{2}\right) t_{1} / \hbar} e^{i\left(E_{3}-E_{4}\right) t_{2} / \hbar}\left[\hat{a}_{L n_{1}}^{\dagger}\left(E_{1}\right) \hat{a}_{L n_{1}}\left(E_{2}\right), \hat{b}_{L n_{2}}^{\dagger}\left(E_{3}\right) \hat{b}_{L n_{2}}\left(E_{4}\right)\right]
$$

and applying the commutation relations as given in Eq. (2) we find that

$$
\begin{aligned}
{\left[\hat{I}_{L, \text { in }}\left(t_{1}\right), \hat{I}_{L, \text { out }}\left(t_{2}\right)\right]=} & \left(\frac{e}{h}\right)^{2} \sum_{n_{1}, n_{2}} \int d E_{1} d E_{2} d E_{3}\left[e^{i\left(E_{1}-E_{3}\right) t_{1} / \hbar} e^{i\left(E_{3}-E_{2}\right) t_{2} / \hbar} \hat{a}_{L n_{1}}^{\dagger}\left(E_{1}\right) s_{L L ; n_{1} n_{2}}^{\dagger}\left(E_{3}\right) \hat{b}_{L n_{2}}\left(E_{2}\right)\right. \\
& \left.-e^{i\left(E_{3}-E_{2}\right) t_{1} / \hbar} e^{i\left(E_{1}-E_{3}\right) t_{2} / \hbar} \hat{b}_{L n_{2}}^{\dagger}\left(E_{1}\right) s_{L L ; n_{2} n_{1}}\left(E_{3}\right) \hat{a}_{L n_{1}}\left(E_{2}\right)\right] .
\end{aligned}
$$

Integrating over all energies we obtain

$$
\left[\hat{I}_{L, \text { in }}\left(t_{1}\right) \hat{I}_{L, \text { out }}\left(t_{2}\right)\right]=h e^{2} \sum_{n_{1}, n_{2}}\left[\hat{a}_{L n_{1}}^{\dagger}\left(t_{1}\right) s_{L L ; n_{1} n_{2}}^{\dagger}\left(t_{2}-t_{1}\right) \hat{b}_{L n_{2}}\left(t_{2}\right)-\hat{b}_{L n_{2}}^{\dagger}\left(t_{2}\right) s_{L L ; n_{2} n_{1}}\left(t_{2}-t_{1}\right) \hat{a}_{L n_{1}}\left(t_{1}\right)\right] .
$$


According to Eq. (11) the commutator (14) vanishes identically if $t_{1}$ is a later instant of time than $t_{2}{ }^{21}$ We thus conclude that

$$
\left[\hat{I}_{L, \text { in }}\left(t_{1}\right), \hat{I}_{L, \text { out }}\left(t_{2}\right)\right] \propto \theta\left(t_{2}-t_{1}\right) .
$$

We obtain the commutation relations for $\hat{I}_{L, \text { in }}\left(t_{1}\right)$ and $\hat{I}_{L, \text { in }}\left(t_{2}\right)$, and for $\hat{I}_{L, \text { out }}\left(t_{1}\right)$ and $\hat{I}_{L, \text { out }}\left(t_{2}\right)$ using the same procedure: both these vanish identically,

$$
\left[\hat{I}_{L, \text { in }}\left(t_{1}\right), \hat{I}_{L, \text { in }}\left(t_{2}\right)\right]=0
$$

and

$$
\left[\hat{I}_{L, \text { out }}\left(t_{1}\right), \hat{I}_{L, \text { out }}\left(t_{2}\right)\right]=0
$$

These commutation relations have important consequences for the calculation of time-ordered correlation functions involving the operators $\hat{I}_{\text {in }}(t)$ and $\hat{I}_{\text {out }}(t)$, as we will now show.

\section{Time-ordered correlation functions}

We denote the time ordering of operators by $T\left[A\left(t_{1}\right) B\left(t_{2}\right) C\left(t_{3}\right) \cdots\right]$, where the operators appear in descending order of times, and the antitime ordering by $\widetilde{T}\left[A\left(t_{1}\right) B\left(t_{2}\right) C\left(t_{3}\right) \cdots\right]$, with the opposite order of times. Specifically, making use of Eqs. (15)-(17), we find the following operator identities:

$$
\begin{gathered}
T\left[\hat{I}_{\text {in }}\left(t_{1}\right) \hat{I}_{\text {in }}\left(t_{2}\right)\right]=\hat{I}_{\text {in }}\left(t_{1}\right) \hat{I}_{\text {in }}\left(t_{2}\right), \\
T\left[\hat{I}_{\text {out }}\left(t_{1}\right) \hat{I}_{\text {out }}\left(t_{2}\right)\right]=\hat{I}_{\text {out }}\left(t_{1}\right) \hat{I}_{\text {out }}\left(t_{2}\right), \\
T\left[\hat{I}_{\text {in }}\left(t_{1}\right) \hat{I}_{\text {out }}\left(t_{2}\right)\right]=\hat{I}_{\text {out }}\left(t_{2}\right) \hat{I}_{\text {in }}\left(t_{1}\right), \\
T\left[\hat{I}_{\text {out }}\left(t_{1}\right) \hat{I}_{\text {in }}\left(t_{2}\right)\right]=\hat{I}_{\text {out }}\left(t_{1}\right) \hat{I}_{\text {in }}\left(t_{2}\right) .
\end{gathered}
$$

One therefore concludes ${ }^{21}$ that time ordering a product of directed current operators corresponds to an ordering in which all the out currents $\hat{I}_{\text {out }}$ are placed to the left of the in currents $\hat{I}_{\text {in }}$.

As an example, let us consider the two lowest timeordered correlation functions. Using $\hat{I}(t)=\hat{I}_{\text {in }}(t)-\hat{I}_{\text {out }}(t)$, one obtains

$$
\begin{aligned}
T\left[\hat{I}\left(t_{1}\right) \hat{I}\left(t_{2}\right)\right]= & \hat{I}_{\text {in }}\left(t_{1}\right) \hat{I}_{\text {in }}\left(t_{2}\right)-\hat{I}_{\text {out }}\left(t_{2}\right) \hat{I}_{\text {in }}\left(t_{1}\right)-\hat{I}_{\text {out }}\left(t_{1}\right) \hat{I}_{\text {in }}\left(t_{2}\right) \\
& +\hat{I}_{\text {out }}\left(t_{1}\right) \hat{I}_{\text {out }}\left(t_{2}\right)
\end{aligned}
$$

and

$$
\begin{aligned}
T\left[\hat{I}\left(t_{1}\right) \hat{I}\left(t_{2}\right) \hat{I}\left(t_{3}\right)\right]= & \hat{I}_{\text {in }}\left(t_{1}\right) \hat{I}_{\text {in }}\left(t_{2}\right) \hat{I}_{\text {in }}\left(t_{3}\right)-\hat{I}_{\text {out }}\left(t_{3}\right) \hat{I}_{\text {in }}\left(t_{1}\right) \hat{I}_{\text {in }}\left(t_{2}\right) \\
& -\hat{I}_{\text {out }}\left(t_{2}\right) \hat{I}_{\text {in }}\left(t_{1}\right) \hat{I}_{\text {in }}\left(t_{3}\right)-\hat{I}_{\text {out }}\left(t_{1}\right) \hat{I}_{\text {in }}\left(t_{2}\right) \hat{I}_{\text {in }}\left(t_{3}\right) \\
& +\hat{I}_{\text {out }}\left(t_{2}\right) \hat{I}_{\text {out }}\left(t_{3}\right) \hat{I}_{\text {in }}\left(t_{1}\right)+\hat{I}_{\text {out }}\left(t_{1}\right) \hat{I}_{\text {out }}\left(t_{3}\right) \hat{I}_{\text {in }}\left(t_{2}\right) \\
& +\hat{I}_{\text {out }}\left(t_{1}\right) \hat{I}_{\text {out }}\left(t_{2}\right) \hat{I}_{\text {in }}\left(t_{3}\right) \\
& -\hat{I}_{\text {out }}\left(t_{1}\right) \hat{I}_{\text {out }}\left(t_{2}\right) \hat{I}_{\text {out }}\left(t_{3}\right) .
\end{aligned}
$$

For the ordered $n$-current correlation function, the number of terms containing $p$ out currents and $n-p$ in currents is just the binomial factor $n ! /[p !(n-p) !]$. The sign of such a term is $(-1)^{p}$. The antitime ordering $\widetilde{T}$ can be dealt with analogously, but here the in and out currents are ordered oppositely: all the out currents stand to the right of the in currents.

The important point here, and one of the central conclusions of Ref. 21, is that using in-out ordering one gets rid of the cumbersome limits of time integration, normally present in time-ordered expressions. This will enable us in the following to straightforwardly calculate Fourier transforms and hence directly obtain the frequency-dependent spectral functions of the relevant correlation functions. Moreover, the idea of ordering currents using the in-out formalism is quite natural in scattering theory.

\section{IN-OUT THREE-CURRENT SPECTRAL FUNCTIONS}

\section{A. General results}

We now turn to consider various three-current correlation functions of the form $\left\langle\delta \hat{I}_{L, x}\left(t_{1}\right) \delta \hat{I}_{L, y}\left(t_{2}\right) \delta \hat{I}_{L, z}\left(t_{3}\right)\right\rangle$, where $x, y$, and $z$ refer to the directed component of the current, either in or out, and $\delta \hat{I}=\hat{I}-\langle\hat{I}\rangle$. In the time-independent case, they can be expressed using the Fourier transform given by

$$
\begin{aligned}
\left\langle\delta \hat{I}_{L, x}\left(t_{1}\right) \delta \hat{I}_{L, y}\left(t_{2}\right) \delta \hat{I}_{L, z}\left(t_{3}\right)\right\rangle \equiv & \int \frac{d \omega_{1}}{2 \pi} e^{-i \omega_{1}\left(t_{1}-t_{2}\right)} \int \frac{d \omega_{2}}{2 \pi} \\
& \times e^{-i \omega_{2}\left(t_{2}-t_{3}\right)} S_{x y z}\left(\omega_{1}, \omega_{2}\right),
\end{aligned}
$$

where $S_{x y z}\left(\omega_{1}, \omega_{2}\right)$ are the corresponding three-current spectral functions. (Note that another convention is to take the transform with respect to $t_{1}-t_{2}$ and $t_{1}-t_{3}$, which leads to slightly redefined parametrization of the spectral functions.) Specializing to the case of equilibrium reservoirs, the spectral functions $S_{x y z}\left(\omega_{1}, \omega_{2}\right)$ are obtained by applying Wick's theorem; we refer the reader to Appendix A for details. Specifically, we present results for the three-current spectral functions in the general case of an arbitrary energydependent scattering matrix in Table II of Appendix B, and for energy-independent scattering in Table III in the same appendix. Here we just note that for the particular case of $S_{\text {in,in,in }}$, the energy integral contains Fermi functions of only one reservoir, and its value vanishes then identically. This is due to the fact that the in-in-in term does not contain the possibly energy-dependent scattering matrix. Spectral functions containing two in-currents also only depend on the Fermi function of the left reservoir, but the energydependence of the scattering matrix may render the integrals nonzero. Such terms, however, vanish in the case of energyindependent scattering so that four spectral functions out of the eight are identically zero. The four remaining spectra at zero temperature are depicted in Fig. 2 as functions of the two frequencies $\omega_{1}$ and $\omega_{2}$.

\section{B. Limiting cases of in-out-ordered spectral functions}

Although the true advantage of in-out ordering comes when dealing with general correlation functions, we demon- 

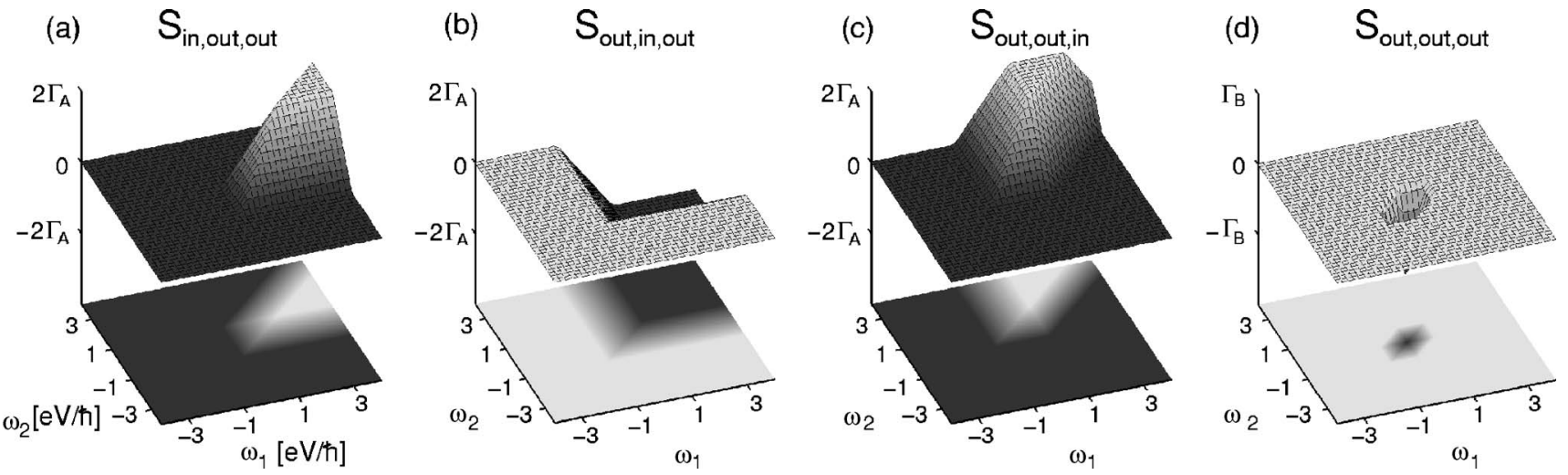

FIG. 2. The nonvanishing contributions to zero-temperature three-current spectral functions $S_{\text {in,out,out, }}, S_{\text {out,in,out }}, S_{\text {out,out,in }}$, and $S_{\text {out,out,out }}$ plotted against the frequencies $\omega_{1}$ and $\omega_{2}$. The first three of these have values between 0 and $\pm 2 \Gamma_{A}= \pm 2 e V \frac{e^{3}}{h} \Sigma_{n} T_{n}\left(1-T_{n}\right)$ while the last one has the extreme value of $-\Gamma_{B}=-e V \frac{e^{3}}{h} \Sigma_{n} T_{n}\left(1-T_{n}\right)\left(1-2 T_{n}\right)$ at the origin and it vanishes at high frequencies $\left|\omega_{1}\right|,\left|\omega_{2}\right| \gg e V / \hbar$.

strate here that it also provides a straightforward way to obtain the spectral functions in some special cases which have been discussed in literature already earlier. In particular, we investigate here the case of energy-independent scattering in the limiting cases in terms of temperature, voltage, and the two frequencies.

As mentioned above, in the case of energy-independent scattering only four three-current spectral functions out of eight possible ones remain nonzero. At zero frequencies $\omega_{1}$ $=\omega_{2}=0$, only $S_{\text {out,in,out }}$ and $S_{\text {out,out,out }}$ are finite, with their values given by

$$
\begin{gathered}
S_{\text {out,in,out }}=-e V \frac{e^{3}}{h} \sum_{n} T_{n}\left(1-T_{n}\right) \equiv-\Gamma_{A}, \\
S_{\text {out }, \text { out }, \text { out }}=-e V \frac{e^{3}}{h} \sum_{n} T_{n}\left(1-T_{n}\right)\left(1-2 T_{n}\right) \equiv-\Gamma_{B},
\end{gathered}
$$

where $\Gamma_{A}=e^{2} G V F_{2}$ and $\Gamma_{B}=e^{2} G V F_{3}$ are expressed in terms of the conductance, $G=\frac{e^{2}}{h} \Sigma_{n} T_{n}$, and the Fano factors of the second and third order $F_{2}=\Sigma_{n} T_{n}\left(1-T_{n}\right) / \Sigma_{n} T_{n}$ and $F_{3}$ $=\Sigma_{n} T_{n}\left(1-T_{n}\right)\left(1-2 T_{n}\right) / \Sigma_{n} T_{n}$. The transmission eigenvalues $\left\{T_{n}\right\}$ are the eigenvalues of the transmission matrix $t^{\dagger} t$. In the high-frequency limit $\left|\omega_{1}\right|,\left|\omega_{2}\right| \gg e V / \hbar$, the nonvanishing terms are in turn $S_{\text {in,out,out }}$ and $S_{\text {out,out,in, }}$, whose values equal $2 \Gamma_{A}$ in the second $\left(\omega_{1}, \omega_{2} \gtrsim 0, \omega_{1} \lesssim \omega_{2}\right)$ and first $\left(\omega_{1}, \omega_{2} \gtrsim 0\right.$, $\left.\omega_{1} \gtrsim \omega_{2}\right)$ octants, respectively, and $S_{\text {out,in,out }}=-2 \Gamma_{A}$ in the first quadrant $\left(\omega_{1}, \omega_{2} \gtrsim 0\right)$.

At finite temperatures such that $\hbar\left|\omega_{1}\right|, \hbar\left|\omega_{2}\right| \ll k_{B} T$, the spectral functions become independent of $\omega_{1}$ and $\omega_{2}$, and the nonvanishing ones are given by

$$
\begin{gathered}
S_{\text {in,out,out }}=S_{\text {out }, \text { out }, \text { in }}=\frac{1}{3} \Gamma_{A}, \\
S_{\text {out }, \text { in out }}=-\frac{2}{3} \Gamma_{A} .
\end{gathered}
$$

\section{DIFFERENT PHYSICAL DETECTOR SCHEMES}

An arbitrary three-current correlation function can always be decomposed into a sum of various in-out-ordered spectral functions of the type of Eq. (21), whose properties are, at least in principle, known. We will illustrate the usefulness of this decomposition scheme now for various examples of three-current correlation functions which have appeared in the literature. For simplicity we assume energy-independent scattering such that definite results can be obtained for three specific examples. We will first consider accumulated charge by a fictitious spin detector, ${ }^{16}$ which directly depends on the Keldysh-ordered correlation functions, and we use the in-out three-current spectral functions to evaluate time-dependent third cumulant of the charge distribution. We also compare this with current statistics derived from an unordered generating function and relate it to some of the results earlier appeared in the literature. The second example is a classical detector which would correspond to the standard fully symmetrized three-current correlation function, ${ }^{28}$ and finally we briefly discuss a partially time-ordered correlation function that appears when the time evolution of the density matrix of a multilevel quantum detector is considered, coupled to a non-Gaussian noise source. ${ }^{29,30}$

\section{A. Third cumulant of FCS}

The third cumulant of the full-counting statistics, i.e., the first correction term describing the deviation from the Gaussian distribution of the charge $q$ transported through the conductor during a time $t$, has been introduced in Refs. 15 and 16. The detector responds to the transmitted current via the cumulant-generating function

$$
\chi(\lambda)=\left\langle\widetilde{T} \exp \left[\frac{i \lambda}{2 e} \int_{0}^{t} d t^{\prime} \hat{I}_{S}\left(t^{\prime}\right)\right] T \exp \left[\frac{i \lambda}{2 e} \int_{0}^{t} d t^{\prime} \hat{I}_{S}\left(t^{\prime}\right)\right]\right\rangle,
$$

where $\lambda$ is the coupling constant (the counting field) between the current and the detector, and the cumulants of the transmitted charge are given by

$$
\left\langle\left\langle q^{p}\right\rangle\right\rangle=\left.e^{p} \frac{d^{p} \log \chi(\lambda)}{d(i \lambda)^{p}}\right|_{\lambda=0} .
$$

The expression for the third cumulant is then given by 


$$
\left\langle\left\langle q^{3}\right\rangle\right\rangle=\int_{0}^{t} d t_{1} \int_{0}^{t} d t_{2} \int_{0}^{t} d t_{3} S_{\mathrm{K}}^{(3)}\left(t_{1}, t_{2}, t_{3}\right)
$$

where the correlation function

$$
\begin{aligned}
S_{\mathrm{K}}^{(3)}\left(t_{1}, t_{2}, t_{3}\right)= & \frac{1}{8}\left\langle\widetilde{T}\left[\hat{I}_{L}\left(t_{1}\right) \hat{I}_{L}\left(t_{2}\right) \hat{I}_{L}\left(t_{3}\right)\right]+T\left[\hat{I}_{L}\left(t_{1}\right) \hat{I}_{L}\left(t_{2}\right) \hat{I}_{L}\left(t_{3}\right)\right]\right. \\
& \left.+3 \widetilde{T}\left[\hat{I}_{L}\left(t_{1}\right) \hat{I}_{L}\left(t_{2}\right)\right] \hat{I}_{L}\left(t_{3}\right)+3 \hat{I}_{L}\left(t_{1}\right) T\left[\hat{I}_{L}\left(t_{2}\right) \hat{I}_{L}\left(t_{3}\right)\right]\right\rangle \\
& -3\left\langle\hat{I}_{L}\left(t_{1}\right)\right\rangle\left\langle\hat{I}_{L}\left(t_{2}\right) \hat{I}_{L}\left(t_{3}\right)\right\rangle+2\left\langle\hat{I}_{L}\left(t_{1}\right)\right\rangle\left\langle\hat{I}_{L}\left(t_{2}\right)\right\rangle
\end{aligned}
$$

$$
\left.\times \hat{I}_{L}\left(t_{3}\right)\right\rangle
$$

has the Keldysh-contour time ordering. Here, in the second last term, the time and antitime orderings of $t_{2}$ and $t_{3}$ can be made to disappear since $\int\left\{\widetilde{T}\left[\hat{I}_{L}\left(t_{2}\right) \hat{I}_{L}\left(t_{3}\right)\right]\right.$ $\left.+T\left[\hat{I}_{L}\left(t_{2}\right) \hat{I}_{L}\left(t_{3}\right)\right]\right\} d t_{2} d t_{3}=2 \int \hat{I}_{L}\left(t_{2}\right) \hat{I}_{L}\left(t_{3}\right) d t_{2} d t_{3}$.

Using the operator relations given by Eqs. (19) and (20), together with their anti-time-ordered counterparts, and regrouping the current operators into deviation operators $\delta \hat{I}_{\text {in,out }}(t) \equiv \hat{I}_{\text {in,out }}(t)-\left\langle\hat{I}_{\text {in,out }}(t)\right\rangle$, enables one to express this particular correlation function as

$$
\begin{aligned}
S_{\mathrm{K}}^{(3)}\left(t_{1}, t_{2}, t_{3}\right)= & \left\langle\delta \hat{I}_{L, \text { in }}\left(t_{1}\right) \delta \hat{I}_{L, \text { in }}\left(t_{2}\right) \delta \hat{I}_{L, \text { in }}\left(t_{3}\right)-\frac{3}{4} \delta \hat{I}_{L, \text { in }}\left(t_{1}\right) \delta \hat{I}_{L, \text { in }}\left(t_{2}\right) \delta \hat{I}_{L, \text { out }}\left(t_{3}\right)-\frac{3}{2} \delta \hat{I}_{L, \text { in }}\left(t_{1}\right) \delta \hat{I}_{L, \text { out }}\left(t_{2}\right) \delta \hat{I}_{L, \text { in }}\left(t_{3}\right)\right. \\
& -\frac{3}{4} \delta \hat{I}_{L, \text { out }}\left(t_{1}\right) \delta \hat{I}_{L, \text { in }}\left(t_{2}\right) \delta \hat{I}_{L, \text { in }}\left(t_{3}\right)+\frac{3}{2} \delta \hat{I}_{L, \text { in }}\left(t_{1}\right) \delta \hat{I}_{L, \text { out }}\left(t_{2}\right) \delta \hat{I}_{L, \text { out }}\left(t_{3}\right)+\frac{3}{2} \delta \hat{I}_{L, \text { out }}\left(t_{1}\right) \delta \hat{I}_{L, \text { out }}\left(t_{2}\right) \delta \hat{I}_{L, \text { in }}\left(t_{3}\right) \\
& \left.-\delta \hat{I}_{L, \text { out }}\left(t_{1}\right) \delta \hat{I}_{L, \text { out }}\left(t_{2}\right) \delta \hat{I}_{L, \text { out }}\left(t_{3}\right)\right\rangle .
\end{aligned}
$$

Each term here can now be expressed in terms of the Fourier transform of the spectral function, Eq. (21), and the time integrals of Eq. (26) may be carried out explicitly. This results in

$$
\begin{aligned}
\left\langle\left\langle q^{3}\right\rangle\right\rangle= & 2 \int \frac{d \omega_{1}}{2 \pi} \int \frac{d \omega_{2}}{2 \pi} S_{\mathrm{K}}^{(3)} \\
& \times\left(\omega_{1}, \omega_{2}\right) \frac{\sin \left(\omega_{2} t\right)-\sin \left(\omega_{1} t\right)+\sin \left[\left(\omega_{1}-\omega_{2}\right) t\right]}{\omega_{1} \omega_{2}\left(\omega_{1}-\omega_{2}\right)},
\end{aligned}
$$

where, for this particular ordering, we have

$$
\begin{aligned}
S_{\mathrm{K}}^{(3)}\left(\omega_{1}, \omega_{2}\right)= & -\frac{3}{4} S_{\mathrm{in}, \mathrm{in}, \mathrm{out}}\left(\omega_{1}, \omega_{2}\right)-\frac{3}{2} S_{\mathrm{in}, \mathrm{out}, \mathrm{in}}\left(\omega_{1}, \omega_{2}\right) \\
& -\frac{3}{4} S_{\text {out,in,in }}\left(\omega_{1}, \omega_{2}\right)+\frac{3}{2} S_{\mathrm{in}, \mathrm{out}, \mathrm{out}}\left(\omega_{1}, \omega_{2}\right) \\
& +\frac{3}{2} S_{\text {out }, \text { out }, \text { in }}\left(\omega_{1}, \omega_{2}\right)-S_{\text {out }, \text { out }, \text { out }}\left(\omega_{1}, \omega_{2}\right) .
\end{aligned}
$$

This result is plotted in Fig. 3(a) for energy-independent scattering at zero temperature. Note that the multiplier of each term in the sum above is obtained with the help of the binomial distribution. The particular ordering for current operators, similar to that in Eq. (27), determines the final weight of each $x y z$ spectral function.

\section{Asymptotic values of the third cumulant}

The third cumulant of FCS can be evaluated in the limits of both short and long times $t$. For short $t$ the cumulant is determined by the values of $S_{K}^{(3)}\left(\omega_{1}, \omega_{2}\right)$ at large frequencies where $S_{\text {out,out,out }}\left(\omega_{1}, \omega_{2}\right)$ is zero, and

$$
S_{K}^{(3)}\left(\omega_{1}, \omega_{2}\right)=3 e V \frac{e^{3}}{h} \sum_{n} T_{n}\left(1-T_{n}\right)=3 \Gamma_{A}
$$

nearly everywhere in the first quadrant of the $\left(\omega_{1}, \omega_{2}\right)$ plane and zero elsewhere. Therefore, the short-time value of the third cumulant is determined by the $S_{\text {in,out,out }}$ and $S_{\text {out,out,in }}$ spectral functions since only they have nonvanishing highfrequency values. We thus have

$$
\begin{aligned}
\left\langle\left\langle q^{3}\right\rangle\right\rangle \approx & 6 t e V \frac{e^{3}}{h} \sum_{n} T_{n}(1 \\
& \left.-T_{n}\right) \int_{0}^{\infty} \frac{d x_{1}}{2 \pi} \int_{0}^{\infty} \frac{d x_{2}}{2 \pi} \frac{\sin x_{2}-\sin x_{1}+\sin \left(x_{1}-x_{2}\right)}{x_{1} x_{2}\left(x_{1}-x_{2}\right)} \\
= & t e V \frac{e^{3}}{h} \sum_{n} T_{n}\left(1-T_{n}\right)=\Gamma_{A} t .
\end{aligned}
$$

Value of the third cumulant for large $t$ is obtained in a similar manner. As long as $S_{\mathrm{K}}^{(3)}(0,0) \neq 0$, the leading order is given by

$$
\begin{aligned}
\left\langle\left\langle q^{3}\right\rangle\right\rangle= & 2 \int \frac{d \omega_{1}}{2 \pi} \int \frac{d \omega_{2}}{2 \pi} S_{K}^{(3)} \\
& \times\left(\omega_{1}, \omega_{2}\right) \frac{\sin \left(\omega_{2} t\right)-\sin \left(\omega_{1} t\right)+\sin \left[\left(\omega_{1}-\omega_{2}\right) t\right]}{\omega_{1} \omega_{2}\left(\omega_{1}-\omega_{2}\right)} \\
\approx & t S_{K}^{(3)}(0,0) .
\end{aligned}
$$

For $k_{B} T \ll e V$, only $S_{\text {out,out,out }}(0,0)=-\Gamma_{B}$ has a nonvanishing value in $S_{K}^{(3)}$ at $\omega_{1}=\omega_{2}=0$, and the linear growth at long times is then given by

$$
\left\langle\left\langle q^{3}\right\rangle\right\rangle=\Gamma_{B} t
$$



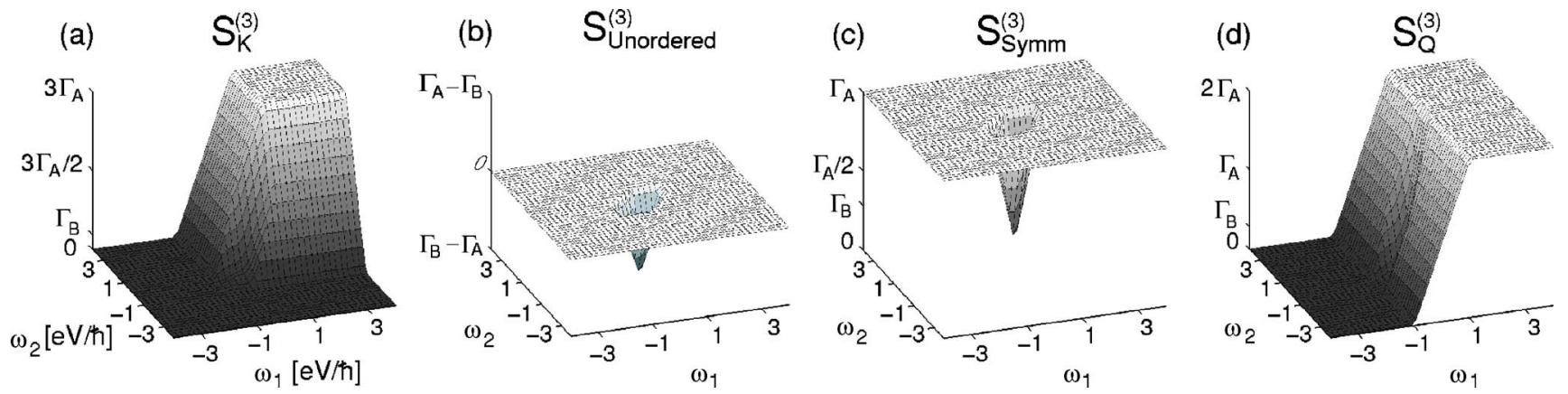

FIG. 3. (Color online) (a) Keldysh-ordered, (b) unordered, (c) symmetrized, and (d) nonsymmetrized spectra at zero temperature. The saturated levels of the spectral functions are all proportional to $\Gamma_{A}$ except for the unordered spectrum, which saturates to zero. For the other spectra the level essentially depends on in which areas of the $\left(\omega_{1}, \omega_{2}\right)$ plane the spectrum has nonzero values. Both $S_{\text {in,out,out }}$ and $S_{\text {out,out,in }}$ vanish at zero frequency, and the spectral functions (a), (c), and (d) are then determined by $\Gamma_{B}=-S_{\text {out,out,out }}$, while the spectrum (b) is given by $\Gamma_{B}-\Gamma_{A}=S_{\text {out,in,out }}-S_{\text {out,out,out }}$, as indicated in the graph. Note that, in the case of energy-independent scattering, the only dependence on the transmission eigenvalues appears through $\Gamma_{A}$ and $\Gamma_{B}$.

while, in the opposite regime, $k_{B} T \gg e V$, the directed threecurrent spectral functions become independent of the frequency arguments, and $S_{K}^{(3)}=e V \frac{e^{3}}{h} \Sigma_{n} T_{n}\left(1-T_{n}\right)$; the long term cumulant is then given by

$$
\left\langle\left\langle q^{3}\right\rangle\right\rangle=\Gamma_{A} t .
$$

Since the Keldysh-ordered spectral function is independent of frequency as long as $\left|\omega_{1}\right|,\left|\omega_{2}\right| \ll k_{B} T / \hbar$, this result holds as long as $t \gg \hbar / k_{B} T$.

Both these results, Eqs. (33) and (34), are in agreement with those presented in Ref. 22, and thus constitute a verification for the correctness of the approach presented. Note in particular that we find $\left\langle\left\langle q^{3}\right\rangle\right\rangle / t=\Gamma_{B} \sim \sum_{n} T_{n}\left(1-T_{n}\right)\left(1-2 T_{n}\right)$ for low temperature. This result has given rise to some discussion in the literature, since Ref. 23 obtained $\left\langle\left\langle q^{3}\right\rangle\right\rangle / t$ $\sim \sum_{n} T_{n}^{2}\left(1-T_{n}\right)$, different from Eq. (33). Several authors ${ }^{24,25}$ subsequently developed methods to analyze frequencydependent three-current correlation functions in order to assess the correctness of Eq. (33). In Ref. 25 an effective action approach together with an involved regularization procedure is used to establish Eq. (33). According to Ref. 24 the fre- quency dependence of $S_{K}$, and hence the result for $\left\langle\left\langle q^{3}\right\rangle\right\rangle$, depends on the actual position of the spin detector with respect to the scatterer. Then, both results for $\left\langle\left\langle q^{3}\right\rangle\right\rangle$ cited above are found, depending on the position of the detector. A drawback is that the specific frequency-dependence of $S_{K}$ postulated in Ref. 24 generally does not conserve current. Let us address the issue here in the framework of the in-outordering technique. The $\Sigma_{n} T_{n}^{2}\left(1-T_{n}\right)$ proportionality is obtained in Ref. 23 by considering a straightforward quantum analogue of the classical generating function, which leads to the cumulant

$$
\begin{aligned}
\left\langle\left\langle q^{3}\right\rangle\right\rangle & =\left\langle\left(\int_{0}^{t} d \tau \delta \hat{I}(\tau)\right)^{3}\right\rangle \\
& =\int_{0}^{t} d t_{1} \int_{0}^{t} d t_{2} \int_{0}^{t} d t_{3} S_{\text {Unordered }}^{(3)}\left(t_{1}, t_{2}, t_{3}\right) .
\end{aligned}
$$

Note that there is no specific time-ordering in this expression. Use of $\delta \hat{I}=\delta \hat{I}_{\text {in }}-\delta \hat{I}_{\text {out }}$ then leads to the entirely unordered correlation function

$$
\begin{aligned}
S_{\text {Unordered }}^{(3)}\left(t_{1}, t_{2}, t_{3}\right)= & \left\langle\delta \hat{I}\left(t_{1}\right) \delta \hat{I}\left(t_{2}\right) \delta \hat{I}\left(t_{3}\right)\right\rangle=\left\langle\delta \hat{I}_{L, \text { in }}\left(t_{1}\right) \delta \hat{I}_{L, \text { in }}\left(t_{2}\right) \delta \hat{I}_{L, \text { in }}\left(t_{3}\right)-\delta \hat{I}_{L, \text { in }}\left(t_{1}\right) \delta \hat{I}_{L, \text { in }}\left(t_{2}\right) \delta \hat{I}_{L, \text { out }}\left(t_{3}\right)-\delta \hat{I}_{L, \text { in }}\left(t_{1}\right) \delta \hat{I}_{L, \text { out }}\left(t_{2}\right) \delta \hat{I}_{L, \text { in }}\left(t_{3}\right)\right. \\
& -\delta \hat{I}_{L, \text { out }}\left(t_{1}\right) \delta \hat{I}_{L, \text { in }}\left(t_{2}\right) \delta \hat{I}_{L, \text { in }}\left(t_{3}\right)+\delta \hat{I}_{L, \text { in }}\left(t_{1}\right) \delta \hat{I}_{L, \text { out }}\left(t_{2}\right) \delta \hat{I}_{L, \text { out }}\left(t_{3}\right)+\delta \hat{I}_{L, \text { out }}\left(t_{1}\right) \delta \hat{I}_{L, \text { out }}\left(t_{2}\right) \delta \hat{I}_{L, \text { in }}\left(t_{3}\right) \\
& \left.+\delta \hat{I}_{L, \text { out }}\left(t_{1}\right) \delta \hat{I}_{L, \text { in }}\left(t_{2}\right) \delta \hat{I}_{L, \text { out }}\left(t_{3}\right)-\delta \hat{I}_{L, \text { out }}\left(t_{1}\right) \delta \hat{I}_{L, \text { out }}\left(t_{2}\right) \delta \hat{I}_{L, \text { out }}\left(t_{3}\right)\right\rangle
\end{aligned}
$$

The corresponding spectrum is given by

$$
\begin{aligned}
S_{\text {Unordered }}^{(3)}\left(\omega_{1}, \omega_{2}\right)= & -S_{\text {in,in,out }}\left(\omega_{1}, \omega_{2}\right)-S_{\text {in,out,in }}\left(\omega_{1}, \omega_{2}\right) \\
& -S_{\text {out }, \text { in ,in }}\left(\omega_{1}, \omega_{2}\right)+S_{\text {in,out }, \text { out }}\left(\omega_{1}, \omega_{2}\right) \\
& +S_{\text {out,in,out }}\left(\omega_{1}, \omega_{2}\right)+S_{\text {out,out,in }}\left(\omega_{1}, \omega_{2}\right) \\
& -S_{\text {out }, \text { out }, \text { out }}\left(\omega_{1}, \omega_{2}\right) ;
\end{aligned}
$$

it is plotted in Fig. 3(b) for zero temperature. Here two terms on the right-hand side of Eq. (37) contribute at zero frequency, namely, $S_{\text {out,in,out }}$ and $S_{\text {out,out,out }}$. For the unordered three current correlator, we thus find that the corresponding third cumulant is given asymptotically (for large $t$ ) by

$$
\left\langle\left\langle q^{3}\right\rangle\right\rangle \approx\left(\Gamma_{B}-\Gamma_{A}\right) t=-t 2 e V \frac{e^{3}}{h} \sum_{n} T_{n}^{2}\left(1-T_{n}\right),
$$


TABLE I. Values of the averaged transmission parameters for three different types of noise sources: a tunnel junction, a chaotic cavity, and a diffusive wire. Here $N$ is the number of transmission channels and $P(T)$ is the distribution function of transmission eigenvalues. In the case of a diffusive wire, $L$ is the length of the wire and $l \ll L$ is the mean free path of electrons.

\begin{tabular}{lccc}
\hline \hline & $\begin{array}{c}\text { Tunnel junction } \\
0 \leq T \ll 1\end{array}$ & $\begin{array}{c}\text { Chaotic cavity } \\
P(T)=\frac{1}{\pi} \frac{1}{\sqrt{T(1-T)}}\end{array}$ & $\begin{array}{c}\text { Diffusive wire } \\
P(T)=\frac{l}{2 L} \frac{1}{T \sqrt{(1-T)}}\end{array}$ \\
\hline$\frac{1}{N}\left\langle\sum_{n} T_{n}\right\rangle$ & $T_{\text {ave }}$ & $\frac{1}{2}$ & $\frac{l}{L}$ \\
$\frac{1}{N}\left\langle\sum_{n} T_{n}\left(1-T_{n}\right)\right\rangle$ & $T_{\text {ave }}$ & $\frac{1}{8}$ & $\frac{1}{3} \frac{l}{L}$ \\
$\frac{1}{N}\left\langle\sum_{n} T_{n}\left(1-T_{n}\right)\left(1-2 T_{n}\right)\right\rangle$ & $T_{\text {ave }}$ & 0 & $\frac{1}{15} \frac{l}{L}$ \\
\hline \hline
\end{tabular}

as found in Ref. 23. We therefore conclude that the difference between this result and Eq. (33) is entirely due to the different ordering properties of the two definitions of $S^{(3)}\left(t_{1}, t_{2}, t_{3}\right)$, given by Eqs. (28) and (36).
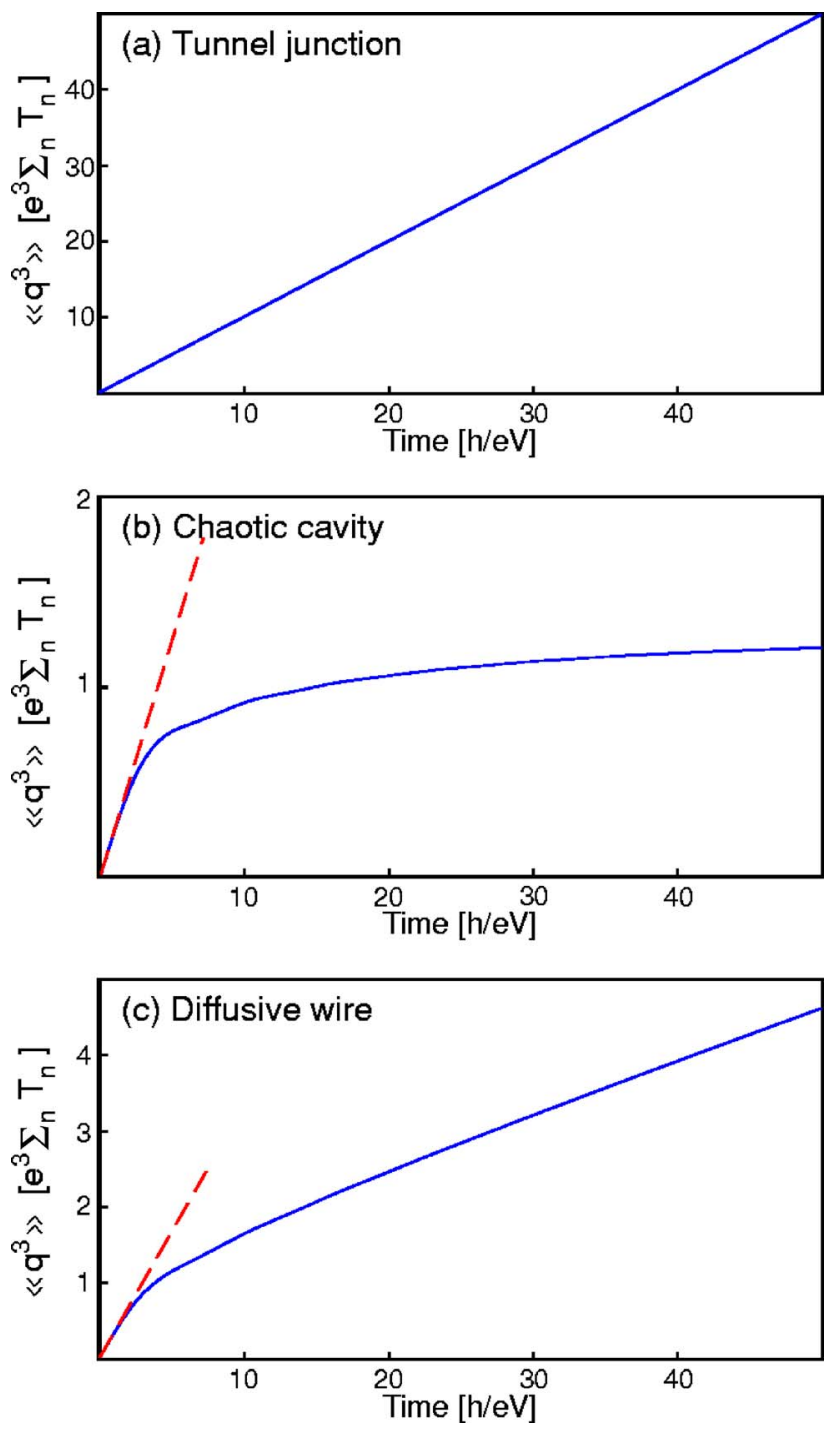

FIG. 4. (Color online) The third cumulant for (a) tunnel junction, (b) chaotic cavity, and (c) diffusive wire at zero temperature. Both the tunnel junction and the diffusive wire show a linear growth at large $t$ due to a nonvanishing zero-frequency value of $S_{\text {out,out,out- }}$

\section{Time-dependent third cumulant in various cases}

We consider separately the time-dependent third cumulant generated by three different kinds of noise sources: a tunnel junction, a chaotic cavity, and a diffusive wire, ${ }^{1}$ in the limit where intrinsic dynamics and interaction effects can be ignored (vanishingly small dwell and charge relaxation times) and scattering can be considered as energy-independent. Then, the transmission properties of these noise sources can be summarized as in Table I.

In an ideal tunnel junction all the transmission probabilities are small $T_{n} \ll 1$ and all the three relevant transmission quantities are equal,

$$
\sum_{n} T_{n}\left(1-T_{n}\right)\left(1-2 T_{n}\right) \approx \sum_{n} T_{n}\left(1-T_{n}\right) \approx \sum_{n} T_{n} \equiv N T_{\mathrm{ave}}
$$

Here $N$ is the number of transport modes penetrating the tunnel barrier. Hence, the linear coefficient of the timedependent third cumulant remains the same in both the small and long time limits. Numerical integration of Eq. (29) demonstrates only this linear increase of the cumulant at all times, as illustrated in Fig. 4(a).

As can be seen from Table I, the transmission probabilities of a chaotic cavity on the other hand are symmetrically distributed between 0 and 1 . Consequently, the coefficient of the out-out-out noise term vanishes and the increase of the third cumulant with time is slower than linear, see Fig. 4(b). Finally, for a diffusive wire the linear growth dominates again for long times, after an initial transient up to several $\hbar / e V$, as can be seen in Fig. 4(c).

\section{B. A fully symmetrized three-current correlation function}

A classical noise detector measures essentially a signal proportional to the symmetrized two-current correlation function

$$
S_{\mathrm{symm}}^{(2)}\left(t_{1}, t_{2}\right)=\frac{1}{2}\left\langle\hat{I}\left(t_{1}\right) \hat{I}\left(t_{2}\right)+\hat{I}\left(t_{2}\right) \hat{I}\left(t_{1}\right)\right\rangle-\langle\hat{I}\rangle^{2} .
$$

It is quite plausible to assume that a classical measurement of the third-order correlations would yield a signal proportional to what is essentially a generalization of Eq. (40), i.e., a fully symmetrized three-current correlation function ${ }^{28}$ 


$$
\begin{aligned}
S_{\text {symm }}^{(3)}\left(t_{1}, t_{2}, t_{3}\right)= & \sum_{i \neq j \neq k=1}^{3}\left\{\frac{1}{16}\left\langle I\left(t_{i}\right) T\left[I\left(t_{j}\right) I\left(t_{k}\right)\right]\right\rangle\right. \\
& +\frac{1}{16}\left\langle\widetilde{T}\left[I\left(t_{i}\right) I\left(t_{j}\right)\right] I\left(t_{k}\right)\right\rangle-\frac{1}{2}\left\langle I\left(t_{i}\right)\right\rangle\left\langle I\left(t_{j}\right) I\left(t_{k}\right)\right\rangle \\
& \left.+\frac{1}{3}\left\langle I\left(t_{i}\right)\right\rangle\left\langle I\left(t_{j}\right)\right\rangle\left\langle I\left(t_{k}\right)\right\rangle\right\} .
\end{aligned}
$$

This correlator is indeed symmetric in all permutations of the time arguments $t_{1}, t_{2}$, and $t_{3}$. We can then immediately rewrite the corresponding spectral function with the help of the in-out-ordering technique as

$$
\begin{aligned}
S_{\text {Symm }}^{(3)}\left(\omega_{1}, \omega_{2}\right)= & \frac{1}{2} S_{\text {in,out }, \text { out }}\left(\omega_{1}, \omega_{2}\right) \\
& +\frac{1}{2} S_{\text {in }, \text { out }, \text { out }}\left(\omega_{2}-\omega_{1},-\omega_{1}\right) \\
& +\frac{1}{2} S_{\text {in,out out }}\left(-\omega_{2}, \omega_{1}-\omega_{2}\right) \\
& +\frac{1}{2} S_{\text {out }, \text { out }, \text { in }}\left(-\omega_{2},-\omega_{1}\right) \\
& +\frac{1}{2} S_{\text {out }, \text { out }, \text { in }}\left(\omega_{1}, \omega_{1}-\omega_{2}\right)+ \\
& \frac{1}{2} S_{\text {out }, \text { out }, \text { in }}\left(\omega_{2}-\omega_{1}, \omega_{2}\right)-S_{\text {out }, \text { out out }}\left(\omega_{1}, \omega_{2}\right) .
\end{aligned}
$$

Here the presence of various combinations of $\omega_{1}$ and $\omega_{2}$ is due to different orderings of the time arguments $t_{1}, t_{2}$, and $t_{3}$, and they also give rise to the hexagonal shape of the spectral function in the $\left(\omega_{1}, \omega_{2}\right)$ plane. This result is plotted in Fig. 3(c) which coincides with the one found in Ref. 28.

Comparing Eqs. (30) and (42), or Figs. 3(a) and 3(c), we see that the symmetrized spectrum is generally quite differ- ent from the Keldysh contour ordered one. Nevertheless, the two coincide in the zero temperature, zero frequency limit such that $S_{\text {symm }}^{(3)}(0,0)=\Gamma_{B}$ and hence corresponds to the usual third cumulant of full counting statistics.

\section{Three-current correlation functions of a multi-level quantum detector}

As it is well known, ${ }^{2,3}$ two-level quantum detector responds to two-current correlators such that the direct transition rate to the higher level (absorption), given by the Fermi golden rule, is normally determined by the nonsymmetrized spectral function

$$
S_{Q}^{(2)}(\omega)=\int_{-\infty}^{\infty} d t e^{i \omega t}\langle\hat{\delta I}(t) \hat{\delta I}(0)\rangle
$$

at the frequency $\omega=-\Delta \omega$, where $\Delta \omega$ is the level spacing. The corresponding relaxation rate (emission) is given by the same spectral function but now at the frequency $+\Delta \omega$. This result can be easily generalized to the case of a multilevel detector.

The next-higher order correction to the transition rate, which includes the effect of transitions via an intermediate state of a multilevel detector, depends, among others, on the three-current spectral function $S_{Q}^{(3)}\left(\omega_{1}, \omega_{2}\right)$, which was recently discussed in Refs. 29 and 30,

$$
\begin{aligned}
S_{Q}^{(3)}\left(t_{1}, t_{2}, t_{3}\right)= & \frac{1}{(2 \pi)^{2}} \int_{-\infty}^{\infty} d \omega_{1} d \omega_{2} e^{-i \omega_{1}\left(t_{1}-t_{2}\right)} e^{-i \omega_{2}\left(t_{2}-t_{3}\right)} \\
& \times S_{Q}^{(3)}\left(\omega_{1}, \omega_{2}\right),
\end{aligned}
$$

where the partially time-ordered three-time current correlation function is

$$
S_{Q}^{(3)}\left(t_{1}, t_{2}, t_{3}\right)=\left\langle\delta I\left(t_{1}\right) T\left[\delta I\left(t_{2}\right) \delta I\left(t_{3}\right)\right]\right\rangle .
$$

We analyze this correlation function here using the in-outordering technique. Expanding in terms of in-out three current correlation functions yields

$$
\begin{aligned}
S_{Q}^{(3)}\left(t_{1}, t_{2}, t_{3}\right)= & \left\langle\delta I_{\text {in }}\left(t_{1}\right) \delta I_{\text {in }}\left(t_{2}\right) \delta I_{\text {in }}\left(t_{3}\right)-\delta I_{\text {in }}\left(t_{1}\right) \delta I_{\text {out }}\left(t_{3}\right) \delta I_{\text {in }}\left(t_{2}\right)-\delta I_{\text {in }}\left(t_{1}\right) \delta I_{\text {out }}\left(t_{2}\right) \delta I_{\text {in }}\left(t_{3}\right)+\delta I_{\text {in }}\left(t_{1}\right) \delta I_{\text {out }}\left(t_{2}\right) \delta I_{\text {out }}\left(t_{3}\right)\right. \\
& \left.-\delta I_{\text {out }}\left(t_{1}\right) \delta I_{\text {in }}\left(t_{2}\right) \delta I_{\text {in }}\left(t_{3}\right)+\delta I_{\text {out }}\left(t_{1}\right) \delta I_{\text {out }}\left(t_{3}\right) \delta I_{\text {in }}\left(t_{2}\right)+\delta I_{\text {out }}\left(t_{1}\right) \delta I_{\text {out }}\left(t_{2}\right) \delta I_{\text {in }}\left(t_{3}\right)-\delta I_{\text {out }}\left(t_{1}\right) \delta I_{\text {out }}\left(t_{2}\right) \delta I_{\text {out }}\left(t_{3}\right)\right\rangle
\end{aligned}
$$

such that the corresponding spectral function is

$$
\begin{aligned}
S_{Q}^{(3)}\left(\omega_{1}, \omega_{2}\right)= & S_{\text {in,out,out }}\left(\omega_{1}, \omega_{2}\right)+S_{\text {out,out,in }}\left(\omega_{1}, \omega_{1}-\omega_{2}\right) \\
& +S_{\text {out }, \text { out }, \text { in }}\left(\omega_{1}, \omega_{2}\right)-S_{\text {out }, \text { out }, \text { out }}\left(\omega_{1}, \omega_{2}\right),
\end{aligned}
$$

see Fig. 3(d). The zero temperature, zero frequency limit of this quantity is given by $S_{Q}^{(3)}(0,0)=\Gamma_{B}$, i.e., it corresponds again to the usual third cumulant of current statistics.

\section{Discussion}

Apart from the unordered spectral function Eq. (37), the various spectral functions discussed so far share many common features at zero temperature. (i) None of them contains the $S_{\text {out,in,out }}$ contribution. (ii) The sum of the terms containing $0,1,2$, and 3 out currents are given by binomial coefficients $(-1)^{k}\left(\begin{array}{l}3 \\ k\end{array}\right)$, where $k$ is the number of out currents. For energy-independent scattering, however, terms with $k=0,1$ vanish. (iii) Regions for which $\left|\omega_{1,2}\right| \geq e V / \hbar$ are only determined by the $k=2$ terms $\left(S_{\text {in,out,out }}\right.$ and $\left.S_{\text {out,out,in }}\right)$ while the 
zero-frequency value is given by the $k=3$ term $\left(S_{\text {out,out,out }}\right)$. (iv) In regions where $\left|\omega_{1,2}\right| \geq e V / \hbar$ the value of the spectral function is either zero or it saturates to a constant, unlike the two-current spectrum which increases linearly. The variously ordered spectral functions differ mainly from each other based on how the "spectral power" is distributed in the $\left(\omega_{1}, \omega_{2}\right)$ plane: the quantum detector noise $S_{Q}^{(3)}$ has twice the

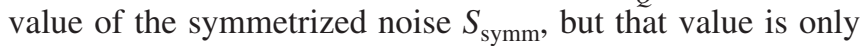
achieved for $\omega_{1}>0$ while the symmetrized noise has the constant level everywhere in the $\left(\omega_{1}, \omega_{2}\right)$ plane, except in the hexagonal area bound within $\left|\omega_{1,2}\right|<e V / \hbar$.

\section{CONCLUSIONS}

In this paper we have considered a formalism that facilitates calculation of time-ordered current correlation functions and applied it to current noise generated by a phasecoherent scatterer. Causality of the real-time representation of the scattering matrix causes products of in- and outcurrent operators $\hat{I}_{\text {in }}\left(t_{1}\right)$ and $\hat{I}_{\text {out }}\left(t_{2}\right)$ to vanish if the in current is taken later than the out current; consequently, time ordering of current operators may be expressed using in-out ordering, in which the out-current operators stand to the left of the in-currents, and vice versa for antitime ordering. The in-out ordering can be directly applied to current correlation functions of arbitrary order, and they can be directly evaluated in the case of thermal reservoirs. If the scattering matrix is, furthermore, energy-independent the correlation functions only depend on the transmission eigenvalues of the scatterer.

It is highly case dependent to which particular current correlator a detector responds, and we evaluate three alternative functions. While a classical noise detector would respond to a fully symmetrized correlator, the spin detector discussed in the case of full counting statistics depends on the Keldysh-contour-ordered correlation function and a multilevel noise detector to a partially or fully time-ordered correlator. We obtain all the answers without cumbersome timeordered integrations.

\section{ACKNOWLEDGMENTS}

We are indebted to M. Büttiker for pointing out Ref. 21 to us as well as for fruitful discussions that motivated us to carry out the work described in this article. We thank Academy of Finland for financial support. F.W.J.H. acknowledges support from the EC-funded ULTI Project, Transnational Access in Programme FP6 (Contract No. RITA-CT-2003505313) and from Institut Universitaire de France.

\section{APPENDIX A: CALCULATION OF THE THREE-CURRENT SPECTRAL FUNCTIONS WITH EQUILIBRIUM RESERVOIRS}

We follow Ref. 17, and obtain all the three-current spectral functions needed by applying Wick's theorem

$$
\begin{aligned}
\left\langle\left(\hat{a}_{k}^{\dagger} \hat{a}_{l}-\left\langle\hat{a}_{k}^{\dagger} \hat{a}_{l}\right\rangle\right)\left(\hat{a}_{m}^{\dagger} \hat{a}_{n}-\left\langle\hat{a}_{m}^{\dagger} \hat{a}_{n}\right\rangle\right)\left(\hat{a}_{p}^{\dagger} \hat{a}_{q}-\left\langle\hat{a}_{p}^{\dagger} \hat{a}_{q}\right\rangle\right)\right\rangle & =\left\langle\hat{a}_{k}^{\dagger} \hat{a}_{q}\right\rangle\left\langle\hat{a}_{l} \hat{a}_{m}^{\dagger}\right\rangle\left\langle\hat{a}_{n} \hat{a}_{p}^{\dagger}\right\rangle-\left\langle\hat{a}_{k}^{\dagger} \hat{a}_{n}\right\rangle\left\langle\hat{a}_{l} \hat{a}_{p}^{\dagger}\right\rangle\left\langle\hat{a}_{m}^{\dagger} \hat{a}_{q}\right\rangle \\
& =\delta_{k q} \delta_{l m} \delta_{n p} f_{k}\left(1-f_{m}\right)\left(1-f_{p}\right)-\delta_{k n} \delta_{l p} \delta_{m q} f_{k} f_{m}\left(1-f_{p}\right) .
\end{aligned}
$$

Next we insert this result into the expression of a three-current correlation function, such as $\left\langle\delta \hat{I}_{L, \text { in }}\left(t_{1}\right) \delta \hat{I}_{L, \text { in }}\left(t_{2}\right) \delta \hat{I}_{L, \text { in }}\left(t_{3}\right)\right\rangle$ :

$$
\begin{aligned}
\left\langle\hat{I}_{L, \text { in }}\left(t_{1}\right) \delta \hat{I}_{L, \text { in }}\left(t_{2}\right) \delta \hat{I}_{L, \text { in }}\left(t_{3}\right)\right\rangle= & \frac{e}{h} \sum_{l} \int_{l} d E_{1} d E_{2} e^{i\left(E_{1}-E_{2}\right) t_{1} / \hbar} \frac{e}{h} \sum_{n} \int d E_{3} d E_{4} e^{i\left(E_{3}-E_{4}\right) t_{2} / \hbar} \frac{e}{h} \sum_{p} \int d E_{5} d E_{6} e^{i\left(E_{5}-E_{6}\right) t_{3} / \hbar} \\
& \times\left\langle[ \hat { a } _ { L , l } ^ { \dagger } ( E _ { 1 } ) \hat { a } _ { L , l } ( E _ { 2 } ) - \langle \hat { a } _ { L , l } ^ { \dagger } ( E _ { 1 } ) \hat { a } _ { L , l } ( E _ { 2 } ) \rangle ] [ \hat { a } _ { L , n } ^ { \dagger } ( E _ { 3 } ) \hat { a } _ { L , n } ( E _ { 4 } ) - \langle \hat { a } _ { L , n } ^ { \dagger } ( E _ { 3 } ) \hat { a } _ { L , n } ( E _ { 4 } ) \rangle ] \left[\hat{a}_{L, p}^{\dagger}\left(E_{5}\right) \hat{a}_{L, p}\left(E_{6}\right)\right.\right. \\
& \left.\left.-\left\langle\hat{a}_{L, p}^{\dagger}\left(E_{5}\right) \hat{a}_{L, p}\left(E_{6}\right)\right\rangle\right]\right\rangle \\
= & -\left(\frac{e}{h}\right)^{3} \sum_{l} \int d E_{1} d E_{3} d E_{5} e^{i\left(E_{1}-E_{5}\right) t_{1} / \hbar} e^{i\left(E_{3}-E_{1}\right) t_{2} / \hbar} e^{i\left(E_{5}-E_{3}\right) t_{3} / \hbar} f_{L}\left(E_{1}\right) f_{L}\left(E_{3}\right)\left[1-f_{L}\left(E_{5}\right)\right] \\
& +\left(\frac{e}{h}\right)^{3} \sum \int d E_{1} d E_{3} d E_{5} e^{i\left(E_{1}-E_{3}\right) t_{1} / \hbar} e^{i\left(E_{3}-E_{5}\right) t_{2} / \hbar} e^{i\left(E_{5}-E_{1}\right) t_{3} / \hbar} f_{L}\left(E_{1}\right)\left[1-f_{L}\left(E_{3}\right)\right]\left[1-f_{L}\left(E_{5}\right)\right] \\
= & \int \frac{d \omega_{1}}{2 \pi} \frac{d \omega_{2}}{2 \pi} e^{-i\left[\omega_{1}\left(t_{1}-t_{2}\right)+\omega_{2}\left(t_{2}-t_{3}\right)\right]} \times \frac{e^{3}}{h} N_{L} \int d E f_{L}(E)\left[1-f_{L}\left(E+\hbar \omega_{1}\right)\right]\left[1-f_{L}\left(E+\hbar \omega_{2}\right)-f_{L}(E\right. \\
& \left.\left.+\hbar \omega_{1}-\hbar \omega_{2}\right)\right]
\end{aligned}
$$

from which we can infer that

$$
S_{\mathrm{in,in,in}}\left(\omega_{1}, \omega_{2}\right)=\frac{e^{3}}{h} N_{L} \int d E f_{L}(E)\left[1-f_{L}\left(E+\hbar \omega_{1}\right)\right]\left[1-f_{L}\left(E+\hbar \omega_{2}\right)-f_{L}\left(E+\hbar \omega_{1}-\hbar \omega_{2}\right)\right],
$$


see Eq. (21). We make next use of the following results valid for Fermi functions:

$$
\begin{gathered}
\int d E f(E)\left[1-f\left(E+\delta E_{1}\right)\right]=\frac{\delta E_{1}}{1-e^{-\beta \delta E_{1}}}, \\
\int d E f(E)\left[1-f\left(E+\delta E_{1}\right)\right]\left[1-f\left(E+\delta E_{2}\right)\right]=\frac{1}{1-e^{-\beta \delta E_{1}}}\left[\frac{\delta E_{2}}{1-e^{-\beta \delta E_{2}}}-\frac{\delta E_{2}-\delta E_{1}}{1-e^{-\beta\left(\delta E_{2}-\delta E_{1}\right)}}\right], \\
\int d E f(E)\left[1-f\left(E+\delta E_{1}\right)\right] f\left(E+\delta E_{2}\right)=\frac{1}{1-e^{-\beta \delta E_{1}}}\left[\delta E_{1}-\frac{\delta E_{2}}{1-e^{-\beta \delta E_{2}}}+\frac{\delta E_{2}-\delta E_{1}}{1-e^{-\beta\left(\delta E_{2}-\delta E_{1}\right)}}\right] .
\end{gathered}
$$

TABLE II. Three-current spectral functions for a general energy-dependent scatterer.

$S_{\text {in,in,in }}\left(\omega_{1}, \omega_{2}\right)=0$

$$
\begin{aligned}
& S_{\text {in,in,out }}\left(\omega_{1}, \omega_{2}\right)=\frac{e^{3}}{h} \int d E\left\{\operatorname{Tr}\left[s_{L L}^{\dagger}\left(E+\hbar \omega_{2}\right) s_{L L}(E)\right] f_{L}(E)\left[1-f_{L}\left(E+\hbar \omega_{1}\right)\right]\left[1-f_{L}\left(E+\hbar \omega_{2}\right)\right]\right. \\
& \left.-\operatorname{Tr}\left[s_{L L}^{\dagger}\left(E+\hbar \omega_{1}\right) s_{L L}\left(E+\hbar \omega_{1}-\hbar \omega_{2}\right)\right] f_{L}(E)\left[1-f_{L}\left(E+\hbar \omega_{1}\right)\right] f_{L}\left(E+\hbar \omega_{1}-\hbar \omega_{2}\right)\right\} \\
& S_{\text {in,out,in }}\left(\omega_{1}, \omega_{2}\right)=\frac{e^{3}}{h} \int d E\left\{\operatorname{Tr}\left[s_{L L}^{\dagger}\left(E+\hbar \omega_{1}\right) s_{L L}\left(E+\hbar \omega_{2}\right)\right] f_{L}(E)\left[1-f_{L}\left(E+\hbar \omega_{1}\right)\right]\left[1-f_{L}\left(E+\hbar \omega_{2}\right)\right]\right. \\
& \left.-\operatorname{Tr}\left[s_{L L}^{\dagger}\left(E+\hbar \omega_{1}-\hbar \omega_{2}\right) s_{L L}(E)\right] f_{L}(E)\left[1-f_{L}\left(E+\hbar \omega_{1}\right)\right] f_{L}\left(E+\hbar \omega_{1}-\hbar \omega_{2}\right)\right\} \\
& S_{\text {out,in,in }}\left(\omega_{1}, \omega_{2}\right)=\frac{e^{3}}{h} \int d E\left\{\operatorname{Tr}\left[s_{L L}^{\dagger}(E) s_{L L}\left(E+\hbar \omega_{1}\right)\right] f_{L}(E)\left[1-f_{L}\left(E+\hbar \omega_{1}\right)\right]\left[1-f_{L}\left(E+\hbar \omega_{2}\right)\right]\right. \\
& \left.-\operatorname{Tr}\left[s_{L L}^{\dagger}(E) s_{L L}\left(E+\hbar \omega_{1}\right)\right] f_{L}(E)\left[1-f_{L}\left(E+\hbar \omega_{1}\right)\right] f_{L}\left(E+\hbar \omega_{1}-\hbar \omega_{2}\right)\right\} \\
& S_{\text {in,out,out }}\left(\omega_{1}, \omega_{2}\right)=\frac{e^{3}}{h} \sum_{\alpha} \int d E\left\{\operatorname{Tr}\left[s_{L L}^{\dagger}\left(E+\hbar \omega_{1}\right) s_{L \alpha}\left(E+\hbar \omega_{2}\right) s_{L \alpha}^{\dagger}\left(E+\hbar \omega_{2}\right) s_{L L}(E)\right] f_{L}(E)\left[1-f_{L}\left(E+\hbar \omega_{1}\right)\right]\left[1-f_{\alpha}\left(E+\hbar \omega_{2}\right)\right]\right. \\
& \left.-\operatorname{Tr}\left[s_{L L}^{\dagger}\left(E+\hbar \omega_{1}\right) s_{L \alpha}\left(E+\hbar \omega_{1}-\hbar \omega_{2}\right) s_{L \alpha}^{\dagger}\left(E+\hbar \omega_{1}-\hbar \omega_{2}\right) s_{L L}(E)\right] f_{L}(E)\left[1-f_{L}\left(E+\hbar \omega_{1}\right)\right] f_{\alpha}\left(E+\hbar \omega_{1}-\hbar \omega_{2}\right)\right\} \\
& S_{\text {out,in,out }}\left(\omega_{1}, \omega_{2}\right)=\frac{e^{3}}{h} \sum_{\alpha} \int d E\left\{\operatorname{Tr}\left[s_{L \alpha}\left(E+\hbar \omega_{1}\right) s_{\alpha L}^{\dagger}(E) s_{L L}\left(E+\hbar \omega_{1}\right) s_{L L}^{\dagger}(E)\right] f_{\alpha}(E)\left[1-f_{L}\left(E+\hbar \omega_{1}\right)\right]\left[1-f_{L}\left(E+\hbar \omega_{2}\right)\right]\right. \\
& \left.-\operatorname{Tr}\left[s_{L L}\left(E+\hbar \omega_{1}\right) s_{L L}^{\dagger}(E) s_{L \beta}\left(E+\hbar \omega_{1}\right) s_{\beta L}^{\dagger}(E)\right] f_{L}(E)\left[1-f_{\beta}\left(E+\hbar \omega_{1}\right)\right] f_{L}\left(E+\hbar \omega_{1}-\hbar \omega_{2}\right)\right\} \\
& S_{\text {out }, \text { out }, \text { in }}\left(\omega_{1}, \omega_{2}\right)=\frac{e^{3}}{h} \sum_{\alpha} \int d E\left\{\operatorname{Tr}\left[s_{L L}^{\dagger}(E) s_{L \alpha}\left(E+\hbar \omega_{1}\right) s_{L \alpha}^{\dagger}\left(E+\hbar \omega_{1}\right) s_{L L}\left(E+\hbar \omega_{2}\right)\right] f_{L}(E)\left[1-f_{\alpha}\left(E+\hbar \omega_{1}\right)\right]\left[1-f_{L}\left(E+\hbar \omega_{2}\right)\right]\right. \\
& \left.-\operatorname{Tr}\left[s_{L \alpha}^{\dagger}(E) s_{L L}\left(E+\hbar \omega_{1}\right) s_{L L}^{\dagger}\left(E+\hbar \omega_{1}-\hbar \omega_{2}\right) s_{L \alpha}(E)\right] f_{\alpha}(E)\left[1-f_{L}\left(E+\hbar \omega_{1}\right)\right] f_{L}\left(E+\hbar \omega_{1}-\hbar \omega_{2}\right)\right\} \\
& S_{\text {out }, \text { out }, \text { out }}\left(\omega_{1}, \omega_{2}\right)=\frac{e^{3}}{h} \sum_{\alpha \beta \gamma} \int \mathrm{d} E\left\{\operatorname{Tr}\left[s_{L \alpha}^{\dagger}(E) s_{L \beta}\left(E+\hbar \omega_{1}\right) s_{L \beta}^{\dagger}\left(E+\hbar \omega_{1}\right) s_{L \gamma}\left(E+\hbar \omega_{2}\right) s_{L \gamma}^{\dagger}\left(E+\hbar \omega_{2}\right) s_{L \alpha}(E)\right] f_{\alpha}(E)\left[1-f_{\beta}\left(E+\hbar \omega_{1}\right)\right][1\right. \\
& \left.-f_{\gamma}\left(E+\hbar \omega_{2}\right)\right]-\operatorname{Tr}\left[s_{L \alpha}^{\dagger}(E) s_{L \beta}\left(E+\hbar \omega_{1}\right) s_{L \beta}^{\dagger}\left(E+\hbar \omega_{1}\right) s_{L \gamma}\left(E+\hbar \omega_{1}-\hbar \omega_{2}\right) s_{L \gamma}^{\dagger}\left(E+\hbar \omega_{1}-\hbar \omega_{2}\right) s_{L \alpha}(E)\right] \times f_{\alpha}(E)[1 \\
& \left.\left.-f_{\beta}\left(E+\omega_{1}\right)\right] f_{\gamma}\left(E+\omega_{1}-\omega_{2}\right)\right\} \text {. }
\end{aligned}
$$


TABLE III. Three-current spectral functions for an energy-independent scatterer.

$S_{\text {in,in,in }}\left(\omega_{1}, \omega_{2}\right)=0$

$S_{\text {in,in,out }}\left(\omega_{1}, \omega_{2}\right)=0$

$S_{\text {in,out,in }}\left(\omega_{1}, \omega_{2}\right)=0$

$S_{\text {out,in,in }}\left(\omega_{1}, \omega_{2}\right)=0$

$S_{\text {in,out }, \text { out }}\left(\omega_{1}, \omega_{2}\right)=\frac{e^{3}}{h} \sum_{n} T_{n}\left(1-T_{n}\right) \int d E\left\{f_{L}(E)\left[1-f_{L}\left(E+\hbar \omega_{1}\right)\right]\left[1-f_{R}\left(E+\hbar \omega_{2}\right)\right]-f_{L}(E)\left[1-f_{L}\left(E+\hbar \omega_{1}\right)\right] f_{R}\left(E+\hbar \omega_{1}-\hbar \omega_{2}\right)\right\}$

$S_{\text {out,in,out }}\left(\omega_{1}, \omega_{2}\right)=\frac{e^{3}}{h} \sum_{n} T_{n}\left(1-T_{n}\right) \int d E\left\{f_{R}(E)\left[1-f_{L}\left(E+\hbar \omega_{1}\right)\right]\left[1-f_{L}\left(E+\hbar \omega_{2}\right)\right]-f_{L}(E)\left[1-f_{R}\left(E+\hbar \omega_{1}\right)\right] f_{L}\left(E+\hbar \omega_{1}-\hbar \omega_{2}\right)\right\}$

$S_{\text {out,out,in }}\left(\omega_{1}, \omega_{2}\right)=\frac{e^{3}}{h} \sum_{n} T_{n}\left(1-T_{n}\right) \int d E\left\{f_{L}(E)\left[1-f_{R}\left(E+\hbar \omega_{1}\right)\right]\left[1-f_{L}\left(E+\hbar \omega_{2}\right)\right]-f_{R}(E)\left[1-f_{L}\left(E+\hbar \omega_{1}\right)\right] f_{L}\left(E+\hbar \omega_{1}-\hbar \omega_{2}\right)\right\}$

$S_{\text {out,out }, \text { out }}\left(\omega_{1}, \omega_{2}\right)=\frac{e^{3}}{h} \sum_{n} T_{n}\left(1-T_{n}\right)^{2} \int d E\left\{f_{L}(E)\left[1-f_{L}\left(E+\hbar \omega_{1}\right)\right]\left[1-f_{R}\left(E+\hbar \omega_{2}\right)-f_{R}\left(E+\hbar \omega_{1}-\hbar \omega_{2}\right)\right]+f_{L}(E)\left[1-f_{R}\left(E+\hbar \omega_{1}\right)\right][1\right.$

$\left.\left.-f_{L}\left(E+\hbar \omega_{2}\right)-f_{L}\left(E+\hbar \omega_{1}-\hbar \omega_{2}\right)\right]+f_{R}(E)\left[1-f_{L}\left(E+\hbar \omega_{1}\right)\right]\left[1-f_{L}\left(E+\hbar \omega_{2}\right)-f_{L}\left(E+\hbar \omega_{1}-\hbar \omega_{2}\right)\right]\right\}$

$+\frac{e^{3}}{h} \sum_{n} T_{n}^{2}\left(1-T_{n}\right) \int d E\left\{f_{L}(E)\left[1-f_{R}\left(E+\hbar \omega_{1}\right)\right]\left[1-f_{R}\left(E+\hbar \omega_{2}\right)-f_{R}\left(E+\hbar \omega_{1}-\hbar \omega_{2}\right)\right]+f_{R}(E)\left[1-f_{L}\left(E+\hbar \omega_{1}\right)\right][1\right.$

$\left.\left.-f_{R}\left(E+\hbar \omega_{2}\right)-f_{R}\left(E+\hbar \omega_{1}-\hbar \omega_{2}\right)\right]+f_{R}(E)\left[1-f_{R}\left(E+\hbar \omega_{1}\right)\right]\left[1-f_{L}\left(E+\hbar \omega_{2}\right)-f_{L}\left(E+\hbar \omega_{1}-\hbar \omega_{2}\right)\right]\right\}$

The integration over energy $E$ can then be performed explicitly. In this particular case of $S_{\text {in,in,in }}\left(\omega_{1}, \omega_{2}\right)$, the energy integral contains Fermi functions of just one reservoir, and therefore its value vanishes:

$$
\left\langle\delta \hat{I}_{L, \text { in }}\left(t_{1}\right) \delta \hat{I}_{L, \text { in }}\left(t_{2}\right) \delta \hat{I}_{L, \text { in }}\left(t_{3}\right)\right\rangle=S_{\text {in, in ,in }}\left(\omega_{1}, \omega_{2}\right)=0 .
$$

This is generally true only for $S_{\text {in,in,in }}$ since it does not depend on the possibly energy-dependent scattering matrix. Spectral functions containing two in currents also have Fermi functions of just the left reservoir, but the energy dependence of the scattering matrix may render the integrals nonzero. Yet in the case of energy-independent scattering such spectral functions vanish.

\section{APPENDIX B: IN-OUT SPECTRAL FUNCTIONS OF THREE CURRENTS}

In Table II all the eight different three-current spectral functions are listed in the general case of energy-dependent scattering and assuming equilibrium reservoirs. The corresponding spectral functions for energy-independent scattering are given in Table III, where $\left\{T_{n}\right\}$ denotes the set of energy-independent eigenvalues of the matrix $t^{\dagger} t$. 
${ }^{1}$ Ya. M. Blanter and M. Büttiker, Phys. Rep. 336, 1 (2000).

${ }^{2}$ R. Aguado and L. P. Kouwenhoven, Phys. Rev. Lett. 84, 1986 (2000).

${ }^{3}$ R. J. Schoelkopf, A. A. Clerk, S. M. Girvin, K. W. Lehnert, and M. H. Devoret, in Ref. 10.

${ }^{4}$ R. Deblock, E. Onac, L. Gurevich, and L. P. Kouwenhoven, Science 301, 203 (2003); P.-M. Billangeon, F. Pierre, H. Bouchiat, and R. Deblock, Phys. Rev. Lett. 96, 136804 (2006).

${ }^{5}$ O. Astafiev, Yu. A. Pashkin, Y. Nakamura, T. Yamamoto, and J. S. Tsai, Phys. Rev. Lett. 93, 267007 (2004).

${ }^{6}$ Y. Naveh, D. V. Averin, and K. K. Likharev, Phys. Rev. Lett. 79, 3482 (1997); Phys. Rev. B 59, 2848 (1999).

${ }^{7}$ K. E. Nagaev, Phys. Rev. B 57, 4628 (1998).

${ }^{8}$ K. E. Nagaev, S. Pilgram, and M. Büttiker, Phys. Rev. Lett. 92, 176804 (2004).

${ }^{9}$ F. W. J. Hekking and J. P. Pekola, Phys. Rev. Lett. 96, 056603 (2006).

${ }^{10}$ Quantum Noise in Mesoscopic Physics, edited by Yu. V. Nazarov (Kluwer, Dordrecht, 2003).

${ }^{11}$ B. Reulet, J. Senzier, and D. E. Prober, Phys. Rev. Lett. 91, 196601 (2003).

${ }^{12}$ Yu. Bomze, G. Gershon, D. Shovkun, L. S. Levitov, and M. Reznikov, Phys. Rev. Lett. 95, 176601 (2005).

${ }^{13}$ S. Gustavsson, R. Leturcq, B. Simovic, R. Schleser, T. Ihn, P. Studerus, K. Ensslin, D. C. Driscoll, and A. C. Gossard, Phys. Rev. Lett. 96, 076605 (2006).

${ }^{14}$ S. Pilgram, K. E. Nagaev, and M. Büttiker, Phys. Rev. B 70,
045304 (2004).

${ }^{15}$ L. S. Levitov and G. B. Lesovik, cond-mat/9401004 (unpublished).

${ }^{16}$ L. S. Levitov, H. Lee, and G. B. Lesovik, J. Math. Phys. 37, 4845 (1996).

${ }^{17}$ M. Büttiker, Phys. Rev. B 46, 12485 (1992).

${ }^{18}$ G. B. Lesovik, Pis'ma Zh. Eksp. Teor. Fiz. 49, 513 (1989) [JETP Lett. 49, 592 (1989)].

${ }^{19}$ B. Yurke and G. P. Kochanski, Phys. Rev. B 41, 8184 (1990).

${ }^{20}$ M. Büttiker, Phys. Rev. Lett. 65, 2901 (1990).

${ }^{21}$ C. W. J. Beenakker and H. Schomerus, Phys. Rev. Lett. 86, 700 (2001).

${ }^{22}$ L. S. Levitov and M. Reznikov, Phys. Rev. B 70, 115305 (2004).

${ }^{23}$ L. S. Levitov and G. B. Lesovik, Pis'ma Zh. Eksp. Teor. Fiz. 55, 534 (1992) [JETP Lett. 55, 555 (1992)].

${ }^{24}$ G. B. Lesovik and N. M. Chtchelkatchev, Pis'ma Zh. Eksp. Teor. Fiz. 77, 464 (2003) [JETP Lett. 77, 393 (2003)].

${ }^{25}$ A. V. Galaktionov, D. S. Golubev, and A. D. Zaikin, Phys. Rev. B 68, 085317 (2003); 68, 235333 (2003).

${ }^{26}$ K. Shepard, Phys. Rev. B 43, 11623 (1991).

${ }^{27}$ M. Büttiker, A. Prêtre, and H. Thomas, Phys. Rev. Lett. 70, 4114 (1993).

${ }^{28}$ D. S. Golubev, A. V. Galaktionov, and A. D. Zaikin, Phys. Rev. B 72, 205417 (2005).

${ }^{29}$ T. Ojanen and T. T. Heikkilä, Phys. Rev. B 73, 020501(R) (2006).

${ }^{30}$ V. Brosco, R. Fazio, F. W. J. Hekking, and J. P. Pekola, Phys. Rev. B 74, 024524 (2006). 